\title{
The Role of the Nuclear Incompressibility in a Relativistic Mean Field Theory for Neutron Stars
}

\author{
V.A. Dexheimer*, C.A.Z. Vasconcellos*, Bardo E.J. Bodmann ${ }^{\dagger}$ and \\ Manfred Dillig** \\ * Instituto de Física, Universidade Federal do Rio Grande do Sul, CEP 91501-970 Porto Alegre, \\ RS, Brazil \\ $\dagger$ Instituto de Física e Matemática, Universidade Federal de Pelotas, CEP 96010-900 Pelotas, Rio \\ Grande do Sul, Brazil \\ **Institut für Theoretische Physik III, der Universität Erlangen-Nürnberg, D91058 Erlangen,
} Germany

\begin{abstract}
We investigate in detail the compression modulus of nuclear matter as a function of the effective nucleon mass. We include consistently in our modelling chemical equilibrium as well as baryon number and electric charge conservation and investigate properties of neutron stars. Among other predictions, we focus on the dependence of the maximum mass of a sequence of neutron stars as a function of the compression modulus and the nucleon effective mass.
\end{abstract}

\section{LAGRANGIAN DENSITY FORMALISM}

The compression modulus has a crucial influence on the dynamics of a broad class of phenomena related to fundamental properties of the equation of state (EoS) for symmetric nuclear matter. From its basic definition, the dynamics of small fluctuations of density around its equilibrium point becomes solely controlled by the compression modulus. There are many attempts in determining the compression modulus of nuclear matter, such as the excitation of the isoscalar giant-monopole resonance[1], non-relativistic approaches within the Hartree-Fock approximation[2] or relativistic formulations based mainly on hadrodynamical models[3]. Values of the compression modulus found vary around $K=(210-220) M e V$ in non-relativistic formulations and $K=(220-300) M e V$ in relativistic approaches. Presently, its value is believed to lie in the range between 220 and $250 \mathrm{MeV}$ [4]. In this work, we focus our analysis on the dependence of the maximum mass of neutron stars as a function of the compression modulus and the nucleon effective mass. The starting point of our investigation is the lagrangian density[5]

$$
\mathscr{L}=\mathscr{L}_{\text {free }}+\sum_{B} \bar{\psi}_{B}\left(g_{\lambda \sigma B}^{\star} \sigma-g_{\beta \omega B}^{\star} \gamma_{\mu} \omega^{\mu}-\frac{1}{2} g_{\gamma \rho B}^{\star} \gamma_{\mu} \tau \cdot \rho^{\mu}\right) \psi_{B},
$$

which describes a system of eight baryons $\left(B=p, n, \Lambda, \Sigma^{-}, \Sigma^{0}, \Sigma^{+}, \Xi^{-}, \Xi^{0}\right)$ coupled to three mesons $(\sigma, \omega, \rho)$ and two leptons $(e, \mu)$. The effective coupling constants parameterize nonlinear self-couplings of the mesons to the baryons. Explicitly they are given by $g_{\sigma \lambda B}^{\star} \equiv m_{\lambda B}^{\star} g_{\sigma} ; g_{\omega \beta B}^{\star} \equiv m_{\beta B}^{\star} g_{\omega} ; g_{\rho \gamma B}^{\star}=m_{\gamma B}^{\star} g_{\rho} ; m_{n B}^{\star} \equiv\left(1+\frac{g_{\sigma} \sigma}{n M_{B}}\right)^{-n} ; n=\lambda, \beta, \gamma$, with $\lambda, \beta$ and $\gamma$ as real and positive phenomenological numbers. From the definition of 
the compression modulus, the pressure of nuclear matter at saturation density vanishes, which yields $K=3 k_{0} d \mu /\left.d k\right|_{k=k_{0}}$. As the chemical potential is a function of $k$ and $\sigma$, its derivative with respect to $k$ is given as $d \mu / d k=(\partial \mu / \partial \sigma)(d \sigma / d k)+\partial \mu / \partial k$. Explicitly we have $\frac{\partial \mu}{\partial k}=m_{\beta}^{* 2} \frac{g_{\omega}^{2}}{m_{\omega}^{2}} \frac{2 k^{2}}{\pi^{2}}+\frac{k}{\sqrt{k^{2}+M^{* 2}}} ; \frac{\partial \mu}{\partial \sigma}=2 m_{\beta}^{*} \frac{g_{\omega}^{2}}{m_{\omega}^{2}} \frac{\partial m_{\beta}^{*}}{\partial \sigma} \frac{2 k^{3}}{3 \pi^{2}}+\frac{M^{*}}{\sqrt{k^{2}+M^{* 2}}} \frac{\partial M^{*}}{\partial \sigma}$.

To find the contribution of the $d \sigma / d k$ term, we consider the role of the $\sigma$ selfconsistently[5], via $f(\sigma, k) \equiv m_{\sigma}^{2} \sigma+F(\sigma) \rho_{s}+G(\sigma) m_{\beta}^{*} \frac{g_{\omega}}{m_{\omega}^{2}} \rho^{2} ; F(\sigma)$ and $G(\sigma)$ are mean-field $\sigma$-dependent functions[5]: $\frac{F(\sigma)}{g_{\sigma}}=-m_{\lambda}^{*}+\frac{g_{\sigma} \sigma}{M}\left(m_{\lambda}^{*}\right)^{(\lambda+1) / \lambda} ; \frac{G(\sigma)}{g_{\sigma} g_{\omega}}=$ $-\frac{\left(m_{\beta}^{*}\right)^{(\beta+1) / \beta}}{M}$, and we apply the condition $f(\sigma, k)=0$ to obtain $\frac{d \sigma}{d k}=\frac{-(\partial f / \partial k)_{\sigma}}{(\partial f / \partial \sigma)_{k}}$, in a explicit form. Combining these results, we finally get an explicit dependence of the compression modulus of nuclear matter on the nucleon effective mass: $K=K_{1}+K_{2}+\frac{K_{3 A} \times K_{3 B}}{K_{3 C}}$, where

$$
\begin{aligned}
& K_{1}=m_{\beta}^{* 2}\left(\frac{g_{\omega}^{2}}{m_{\omega}^{2}}\right) \frac{6 k_{0}^{3}}{\pi^{2}} ; K_{2}=\frac{3 k_{0}^{2}}{\sqrt{k_{0}^{2}+M^{* 2}}} ; \\
& K_{3 A}=-3 k_{0}\left[2\left(\frac{g_{\omega}^{2}}{m_{\omega}^{2}}\right) m_{\beta}^{*}\left(\frac{G(\sigma)}{g_{\sigma} g_{\omega}}\right) \frac{2 k_{0}^{3}}{3 \pi^{2}}+\frac{M^{*}}{\sqrt{k_{0}^{2}+M^{* 2}}}\left(\frac{F(\sigma)}{g_{\sigma}}\right)\right] ; \\
& K_{3 B}=\left[\left(\frac{g_{\sigma}^{2}}{m_{\sigma}^{2}}\right)\left(\frac{F(\sigma)}{g_{\sigma}}\right) \frac{2 k_{0}^{2} M^{*}}{\sqrt{k_{0}^{2}+M^{* 2}}}\right]\left[1+\frac{\left[2 m_{\beta}^{*}\left(\frac{G(\sigma}{g_{\sigma} g_{\omega}}\right)\left(\frac{g_{\omega}^{2}}{m_{\omega}^{2}}\right) \frac{4 k_{0}^{5}}{3 \pi^{4}}\right]}{\left[\frac{F(\sigma)}{g_{\sigma}} \frac{2 k_{0}^{2} M^{*}}{\sqrt{k_{0}^{2}+M^{* 2}}}\right]}\right] ; \\
& +\left(\frac{F(\sigma)}{g_{\sigma}}\right)^{2}\left(\frac{g_{\sigma}^{2}}{m_{\sigma}^{2}}\right)\left(\frac{2}{3 \pi^{2}}\left(\int_{0}^{k_{0}} \frac{1}{\left(k^{2}+M^{* 2}\right)^{1 / 2}} d^{3} k-\int_{0}^{k_{0}^{2}} \frac{M^{*}}{\left(k^{2}+M^{* 2}\right)^{3 / 2}} d^{3} k\right)\right) \\
& +\left(\frac{g_{\omega}^{2}}{m_{\omega}^{2}}\right)\left(\frac{g_{\sigma}^{2}}{m_{\sigma}^{2}}\right) \frac{4 k_{0}^{6}}{9 \pi^{4}}\left[m_{\beta}^{*}\left(\frac{1}{g_{\omega} g_{\sigma}^{2}} \frac{\partial G(\sigma)}{\partial \sigma}\right)+\left(\frac{G(\sigma)}{g_{\sigma} g_{\omega}}\right)^{2}\right] .
\end{aligned}
$$

\section{RESULTS AND CONCLUSIONS}

From the definition of the effective coupling constants, our effective model allows to control the intensity of the scalar, vector and isovector mean-field potentials. Variations of parameters allow to obtain values of $S, V, M^{*}$ and $K$, which correspond to the intermediate regions of values of Walecka, ZM3 and ZM models, for instance. We found the range of possible values for the parameters of the theory is fairly restricted. Due to the form of the new couplings, there occurs a rapid convergence to exponential forms: for $\lambda$ and/or $\beta=\gamma>2$ the results of this model do not strongly differ from the results of the 

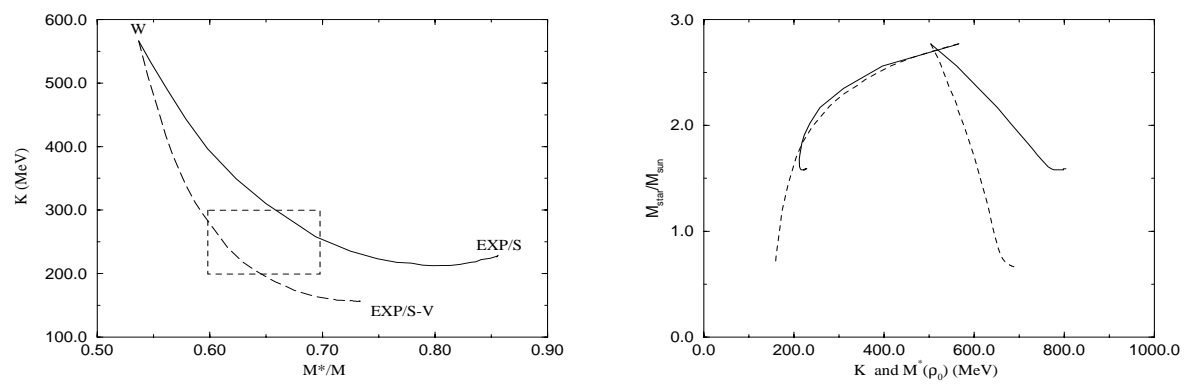

FIGURE 1. Left panel: comparison of the compression modulus $K$ of nuclear matter with the ratio $M^{*} / M$ for the S and S-V cases. The box indicates the range of acceptable values. Right panel: maximum neutron star mass as a function of the compression modulus (left curves) and nucleon non-linear mass at saturation (right curves). The solid lines corresponds to variations of the parameter $\lambda$ keeping the parameters $\beta=\gamma=0$ (S case) and the dashed lines to variations of $\lambda$, keeping $\beta=\gamma=\lambda$ (S-V case).

model with exponential couplings $g_{\sigma \lambda B}^{\star} \stackrel{\lambda \rightarrow \infty}{\longrightarrow} e^{-\frac{g_{\sigma} \sigma}{M_{B}}} g_{\sigma} ; g_{\omega \beta B}^{\star} \stackrel{\beta \rightarrow \infty}{\longrightarrow} e^{-\frac{g \sigma \sigma}{M_{B}}} g_{\omega} ; g_{\rho \gamma B}^{\star} \stackrel{\gamma \rightarrow \infty}{\longrightarrow}$ $e^{-\frac{g \sigma \sigma}{M_{B}}} g_{\rho}$. We consider two cases: i) variations of $\lambda$ keeping $\beta=\gamma=0$; this is case $\mathrm{S}$ (scalar) because the contributions corresponding to $\sigma-\omega$ and $\sigma-\rho$ interactions are not taken into account; it contains the results of the Walecka and ZM models; ii) variations of $\lambda$, keeping $\beta=\gamma=\lambda$; this is case S-V (scalar-vector) because the contributions corresponding to $\sigma-\omega$ and $\sigma-\rho$ interactions are taken into account ; Walecka and ZM3 models belong to this category. (The most simple version of a RMF approach, the Walecka model, belongs to both categories because in this model the $\lambda, \beta$ and $\gamma$ parameters vanish.)

As a novel result (see the figures), we have obtained an explicit dependence of the compression modulus of nuclear matter on the nucleon effective mass. Our results show that a fixed value of the compression modulus corresponds to very close values of the maximal neutron star mass; opposite to that, different values of the star mass correspond to close values of the nucleon effective mass. From these findings, the main conclusion we arrive is that predictions of neutron star properties based mainly on the compression modulus are more model independent than those based on the nucleon effective mass.

\section{REFERENCES}

1. D.H. Youngblood et al., Nucl. Phys. A649 (1999) 49c.

2. J.P. Blaizot et al., Nucl. Phys. A591 (1995) 435.

3. B.D. Serot and J.D. Walecka, Int. Jour. Mod. Phys. E6 (1997) 515.

4. F. Weber, Int. J. Mod. Phys. D (2004) (to be published).

5. A. R. Taurines, C. A. Z. Vasconcellos, M. Malheiro and M. Chiapparini, Phys. Rev. C63 (2001) 1; Mod. Phys. Let. A15 1789 (2000) 1789.

6. J. Piekarewicz, J. Piekarewicz, Phys. Rev. C66 (2002) 034305; nucl-th/0205007 (2002). 
Copyright of AIP Conference Proceedings is the property of American Institute of Physics and its content may not be copied or emailed to multiple sites or posted to a listserv without the copyright holder's express written permission. However, users may print, download, or email articles for individual use. 